\title{
Renal Leiomyosarcoma: Case Report and Review of the Literature
}

\author{
Young Suk Kwon ${ }^{\mathrm{a}}$, Amirali Salmasi ${ }^{\mathrm{b}}$, Christopher Sejong Han ${ }^{\mathrm{b}}$, Jane Date C. Hon ${ }^{\mathrm{c}}$, Eric A. Singer ${ }^{\mathrm{a}}$, b, d
}

\begin{abstract}
Leiomyosarcoma of the kidney is a rare entity, and our understanding of this type of renal sarcomas is limited. A 46-year-old Caucasian male presented with a chief complaint of right flank pain for 1 month. He came to our facility for an additional opinion regarding the management of his renal mass. Computed tomography (CT) of the abdomen showed an enhancing, heterogeneous right renal mass, consistent with the features of renal cell carcinoma (RCC). Robotic-assisted total nephrectomy of the right kidney revealed a tan mass with central necrosis that involved the upper pole of the kidney. Based on gross specimen observation and immunochemical analysis, the patient was diagnosed with high-grade leiomyosarcoma. While the prognosis is poor, radical nephrectomy remains the treatment of choice. The potential benefits of adjuvant therapy should be discussed with selected patients.
\end{abstract}

Keywords: Leiomyosarcoma; Kidney; Adjuvant therapy; Nephrectomy

\section{Introduction}

Leiomyosarcoma of the kidney is an exceedingly uncommon tumor, accounting for only $0.12 \%$ of renal malignancies [1]. It is also known to preferentially affect females in their 60s [2]. Although leiomyosarcoma is not commonly encountered in a clinical setting, the aggressive course of this tumor, its similar-

Manuscript accepted for publication May 01, 2015

aSection of Urologic Oncology, Rutgers Cancer Institute of New Jersey, New Brunswick, NJ, USA

${ }^{b}$ Division of Urology, Rutgers Robert Wood Johnson Medical School, New Brunswick, NJ, USA

'Department of Pathology, Rutgers Robert Wood Johnson Medical School, New Brunswick, NJ, USA

${ }^{\mathrm{d} C}$ Corresponding Author: Eric A. Singer, Section of Urologic Oncology, Rutgers Cancer Institute of New Jersey, Rutgers, The State University of New Jersey, 195 Little Albany Street, New Brunswick, NJ 08903, USA.

Email: eric.singer@rutgers.edu

doi: http://dx.doi.org/10.14740/wjnu214w ity to renal cell carcinoma (RCC), and the potential role of adjuvant therapy mandate our understanding of this pathology. Here we present our case of renal leiomyosarcoma in a male patient along with a review of the literature on this topic.

\section{Case Report}

A 46-year-old man presented to our facility for a second opinion regarding the management of his right renal mass shown in ultrasound and computed tomography (CT) of abdomen and pelvis 10 days prior to his visit. CT scan revealed an enhancing, heterogeneous, $9.5 \mathrm{~cm}$ endophytic right renal mass (Fig. 1). He had microscopic hematuria on urinalysis and suffered from right flank pain of 1 month duration.

His metastatic workup was completed with a chest CT, which showed $3 \mathrm{~mm}$ lung nodules in the left upper lobe and the right lower lobe. Based on the location and characteristics of the kidney mass, the clinical diagnosis of RCC was made. The patient agreed with the surgical management of his renal mass. Transperitoneal robot-assisted total nephrectomy was performed without any complications and with no tumor spillage. The adrenal gland was spared, and margin status was negative.

On the hemisection of the kidney by pathology, a tan mass measuring $8.0 \times 7.5 \times 7.5 \mathrm{~cm}$ was found to occupy the entire upper pole of the kidney. Adjacent ureter, renal vessels and lymph nodes were disease-free with no definitive evidence of lymphovascular invasion. Surgical margins were negative. Upon microscopic evaluation, numerous spindle tumor cells were present adjacent to uninvolved kidney parenchyma (Fig. 2). An interlacing fascicular pattern was observed in groups of spindle cells (Fig. 3). Immunohistochemical analysis revealed that the tumor was positive for smooth muscle actin, caldesmon (Fig. 4), and vimentin, but was negative for S100, BCL2, CAM5.2, CK7, CD34 and desmin.

Based on the tumor differentiation, presence of necrosis, and level of mitosis (Fig. 5), the final diagnosis of high-grade leiomyosarcoma was made. Necrosis was noted. An option of adjuvant chemotherapy was considered and was discussed with the patient to further reduce the risk of micrometastatic disease. The patient was referred to medical oncology for a formal consultation. Postoperatively, the patient is recovering well from the surgery, and a 3-month follow-up visit is scheduled with $\mathrm{CT}$ scan of chest, abdomen, and pelvis for surveillance. 

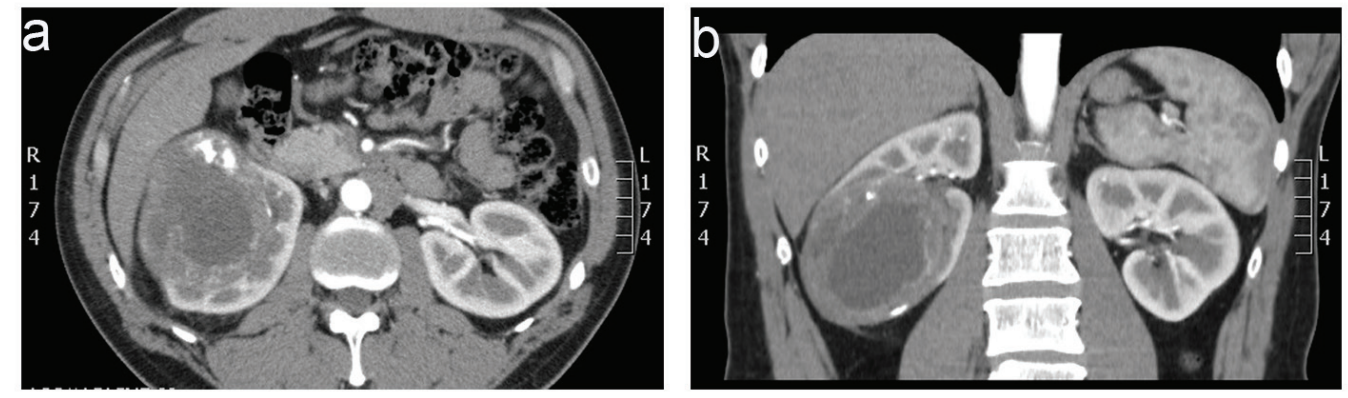

Figure 1. Computed tomography of abdomen and pelvis without contrast prior to surgery showed an enhancing, heterogeneous, $9.5 \mathrm{~cm}$ endophytic right renal mass with mild retroperitoneal lymphadenopathy (a) axial view (b) coronal view.

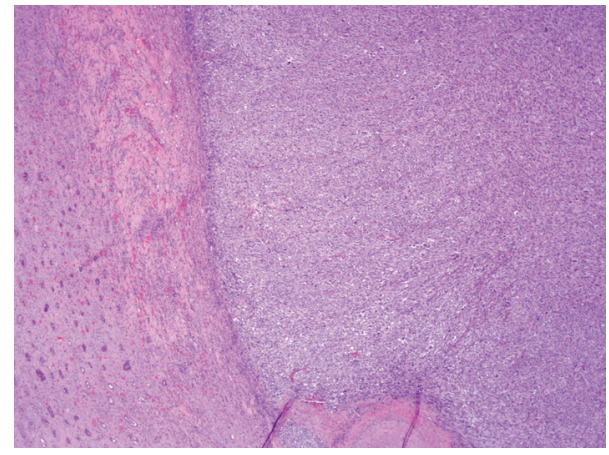

Figure 2. Sharply demarcated, spindle tumor cells are seen adjacent to uninvolved kidney parenchyma (HE, × 40).

\section{Discussion}

Although it is the most common histological subtype and accounts for $50-60 \%$ of total renal sarcomas, leiomyosarcoma is still a remarkably rare tumor [2]. The cause of female predominance is not fully known, but studies suggest that some malignancies are associated with genes located on X chromosomes that escape X-inactivation [3]. In this regard, our male patient makes our case more unique when compared to previous reports (Table 1) [4-9]. In terms of its presenting symptoms, leiomyosarcoma mimics other renal malignancies. Clinical presentations tend to be non-specific as patients

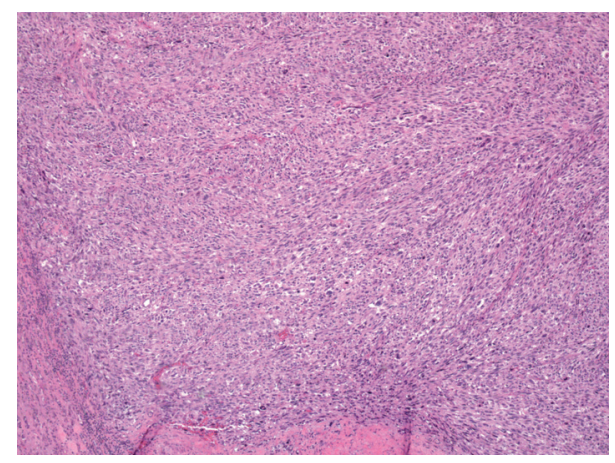

Figure 3. Fascicular arrangement of spindled cells are seen $(\mathrm{HE}, \times$ 100).

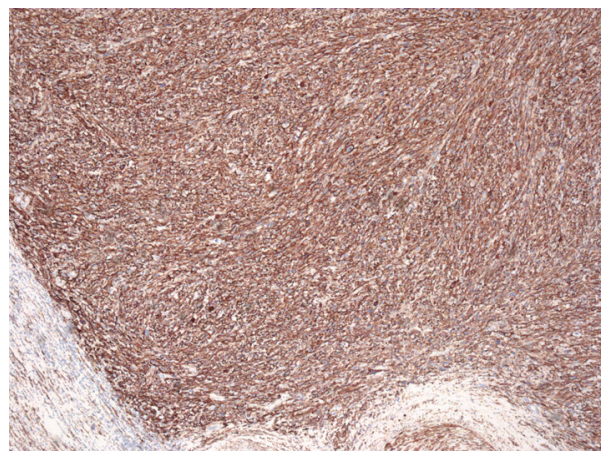

Figure 4. Staining for caldesmon, a smooth muscle marker, shows strong cytoplasmic positivity (× 100).

typically complain of flank pain or abdominal pain and hematuria. One additional challenge in making the correct diagnosis is that conventional imaging is limited as the tumor characteristics seen on imaging may not be sufficient to reliably differentiate leiomyosarcoma from RCC [10]. Therefore, it is not surprising that renal leiomyosarcoma is often mistaken for RCC prior to surgery and subsequent tissue analysis $[4$, 5].

Some of the distinguishing pathologic features of leiomyosarcoma, as opposed to leiomyoma, are the presence of cellular necrosis, nuclear polymorphism, and increased mitotic rate [6]. These are universal characteristics described in the

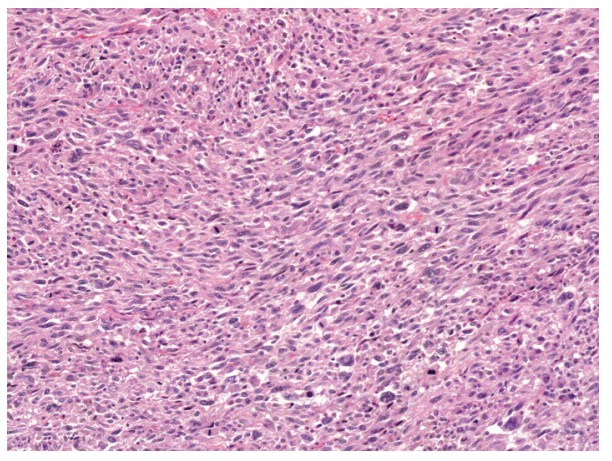

Figure 5. At $\times 200$, numerous mitoses are evident. The nuclei are cigar-shaped with granular eosinophilic cytoplasm. Focal giant cells are present. 


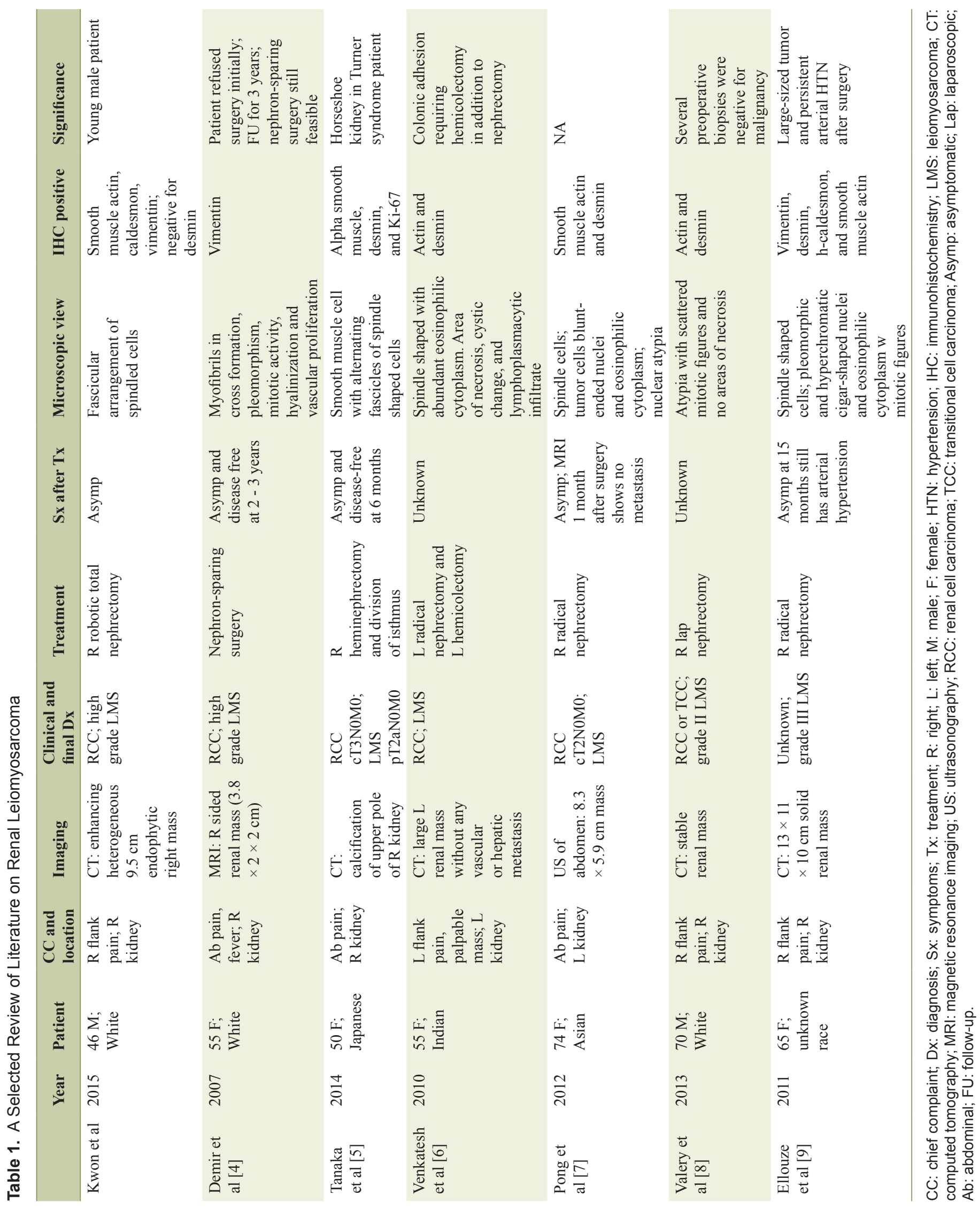


literature. Microscopically, as with other earlier reports, our patient's pathologic specimen revealed spindle cells with areas of necrosis. Although the desmin result was negative in our case, immunohistochemical analysis typically shows positive expressions for smooth muscle actin, calponin, desmin, and h-caldesmon in renal leiomyosarcoma [7] and represents the alteration of the bundled arrangement of cytoskeleton, illustrating the process of the neoplastic transformation [11]. Immunohistochemical examination is also useful to differentiate leiomyosarcoma from sarcomatoid RCC when the distinction can be difficult based solely on histological comparisons [7]. According to the French Federation of Cancer Center classification system, grades are assigned based on differentiation, necrosis and mitosis. Because our patient has a high-grade tumor, he would likely have a poor prognosis with 5-year survival rate less than $40 \%$ [12].

The current treatment of choice for renal leiomyosarcoma is radical nephrectomy [12]. Demir et al reported a case of effective management of leiomyosarcoma with nephron-sparing surgery, but the size of the described patient's renal mass had not changed for 3 years. Hence, the tumor was more likely a low-grade tumor [4]. Considering that a significant proportion of aggressive leiomyosarcomas tend to recur locally, radical nephrectomy is a superior management option that provides a better oncologic control [4].

Although technically challenging, an accurate preoperative diagnosis may be beneficial as neoadjuvant chemotherapy can be utilized to treat potential micrometastasis in leiomyosarcoma [13]. Moreover, adjuvant radiotherapy and chemotherapy may be used in the care of patients with renal leiomyosarcoma. Sharma et al described a low-grade leiomyosarcoma case where the patient received post-operative chemotherapy with mesna, adriamicin, ifosfamide, and dacarbazine regimen and sandwich radiotherapy with a dose of 44 Gy in fractions to the renal bed and adjoining lymphatic area [10]. We advised our patient about the potential benefits of adjuvant chemotherapy despite his negative margin status and the absence of metastasis. Our recommendation was based on his high-risk features including abdominal/retroperitoneal location, size greater than $5 \mathrm{~cm}$, high-grade histology, and presence of necrosis [14]. While both neoadjuvant and adjuvant therapies may benefit a selected group of patients, no randomized control trials have demonstrated their long-term effects and should be individually tailored.

\section{Conclusion}

Primary leiomyosarcoma of the kidney is a rare, but an important tumor that may present similarly to other renal malignancies. Because the presenting symptoms and results from imaging tests do not provide a sufficient ground for accurate and timely diagnosis, a high index of suspicion should be maintained. Although surgical excision is the gold standard treatment for renal leiomyosarcoma, both neoadjuvant and adjuvant treatment modalities should be considered to better accomplish oncologic control. Therefore, patients with renal leiomyosarcoma should be referred for multidisciplinary management (urologic oncology/surgical oncology, medical oncology, and radiation oncology) at an experienced sarcoma center.

\section{Funding}

This work is supported by a grant from the National Cancer Institute (P30CA072720).

\section{Conflict of Interest}

All authors have no direct or indirect conflict of interest with any institution or product.

\section{References}

1. Dhawan S, Chopra P. Primary renal leiomyosarcoma: A diagnostic challenge. Urol Ann. 2012;4(1):48-50.

2. Niceta P, Lavengood RW, Jr., Fernandes M, Tozzo PJ. Leiomyosarcoma of kidney. Review of the literature. Urology. 1974;3(3):270-277.

3. Brown CJ, Greally JM. A stain upon the silence: genes escaping X inactivation. Trends Genet. 2003;19(8):432438.

4. Demir A, Yazici CM, Eren F, Turkeri L. Case report: good prognosis in leiomyosarcoma of the kidney. Int Urol Nephrol. 2007;39(1):7-10

5. Tanaka T, Koie T, Iwabuchi I, Ogasawara M, Kawaguchi T, Ohyama C. Primary leiomyosarcoma of a horseshoe kidney in a woman with Turner syndrome: a case report. BMC Res Notes. 2014; 7:491.

6. Venkatesh K, Lamba Saini M, Niveditha SR, Krishnagiri C, Babu S. Primary leiomyosarcoma of the kidney. Patholog Res Int. 2010;2010:652398.

7. Pong YH, Tsai VFS, Wang SM. Primary leiomyosarcoma of the kidney. Formosan Journal of Surgery. 45(4):124126.

8. Valery JR, Tan W, Cortese C. Renal leiomyosarcoma: a diagnostic challenge. Case Rep Oncol Med. 2013;2013:459282.

9. Ellouze S, Abid N, Kossentini M, Gouiaa N, Charfi S, Mhiri N, Boudawara T. Leiomyosarcoma of the kidney. Clin Genitourin Cancer. 2011;9(1):68-69.

10. Sharma D, Pradhan S, Aryya NC, Shukla VK. Leiomyosarcoma of kidney: a case report with long term result after radiotherapy and chemotherapy. Int Urol Nephrol. 2007;39(2):397-400.

11. Hemmi A, Komiyama A, Ohno S, Fujii Y, Katoh R, Yokoyama A, Kawaoi A. Poorly differentiated desminnegative and vimentin-positive leiomyosarcoma of the stomach examined by the immunohistochemical and quick-freezing and deep-etching methods. Virchows Arch. 1998;432(4):377-383.

12. Deyrup AT, Montgomery E, Fisher C. Leiomyosarcoma of the kidney: a clinicopathologic study. Am J Surg Pathol. 2004;28(2):178-182. 
13. Miller JS, Zhou M, Brimo F, Guo CC, Epstein JI. Primary leiomyosarcoma of the kidney: a clinicopathologic study of 27 cases. Am J Surg Pathol. 2010;34(2):238-242.
14. Dominici A, Mondaini N, Nesi G, Travaglini F, Di Cello V, Rizzo M. Cystic leiomyosarcoma of the kidney: an unusual clinical presentation. Urol Int. 2000;65(4):229-231. 